\title{
Fuentes de Carga Mental en una Muestra de Enfermeros y Técnicos Auxiliares de Enfermería de Madrid (España)
}

\author{
Sources of Mental Workload in a Sample of Nurses and Auxiliary Nursing \\ Technicians from Madrid (Spain)
}

\author{
Susana Rubio-Valdehita ${ }^{1}$ y Isabel Rodrigo-Tapias ${ }^{2}$
}

\begin{abstract}
Resumen
La carga mental representa uno de los principales factores de riesgo psicosocial del trabajo y es causa de importantes problemas en el rendimiento y en la salud de los trabajadores. El objetivo del presente estudio es analizar las fuentes de carga mental referidas por una muestra de profesionales de la enfermería de Madrid (España). Se distinguieron cuatro funciones generales del trabajo: asistencial, administrativa, apoyo a otros facultativos y tareas inherentes a la especialidad médica. La muestra estaba compuesta por 297 trabajadores de hospitales de Madrid y las estimaciones de carga mental para cada función general se obtuvieron mediante el instrumento NASA-TLX. Los resultados muestran que los trabajadores refieren más carga mental en las funciones asistencial y administrativa, y señalan como fuentes de carga más importantes la demanda mental y la temporal. Por último, se señalan algunas conclusiones y recomendaciones de cara a una posible intervención.
\end{abstract}

Palabras clave: carga mental, NASA-TLX, enfermería, salud laboral, riesgos psicosociales

\begin{abstract}
The mental workload is one of the main psychosocial risk factors at work and causes major problems in the performance and health of workers. The aim of this study is to analyze the sources of mental workload reported by a sample of nurses from Madrid (Spain). After an analysis of the tasks were derived four broad job functions: assistance, administrative, support to other personnel and tasks specifically related to medical specialty. The sample consisted of 297 workers in public hospitals in Madrid and mental workload was estimated for each general function using the NASA-TLX scale. The results show that workers report more mental workload on the assistance and administrative functions, and point out as the most important workload sources the mental demand and the temporal demand. Finally, some conclusions and recommendations for a possible intervention are mentioned.
\end{abstract}

Keywords: mental workload, NASA-TLX, nurse, occupational health, psychosocial risks

${ }^{1}$ Facultad de Psicología. Campus de Somosaguas. 28223 Pozuelo de Alarcón, Madrid, España. Tel.:+34913942877. Correo: srubiova@ucm.es

${ }^{2}$ Facultad de Psicología. Campus de Somosaguas. 28223 Pozuelo de Alarcón, Madrid, España. Tel.:+34913942877.

Correo: isabel.rodrigotapias@gmail.com 


\section{Introducción}

En España, la Ley de Prevención de Riesgos Laborales 31/1995 del 8 de noviembre, define las condiciones de trabajo como aquellas características que pueden tener una influencia significativa en la generación de riesgos para la seguridad y salud del trabajador, y obliga a todas las empresas a realizar evaluaciones periódicas de dichos riesgos de manera que se establezcan las medidas de prevención adecuadas. Las características del trabajo referidas a su organización, al contenido del puesto, a la realización de la tarea e incluso las relacionadas con el entorno, y que afectan a la salud de las personas a través de mecanismos psicológicos, constituyen los denominados factores psicosociales de riesgo laboral (Sauter, Murphy, Hurrell, \& Levi, 2001). Los riesgos psicosociales son, por tanto, el resultado de deficiencias en el diseño, la organización o la gestión del trabajo. Estos riesgos pueden producir alteraciones a nivel psicológico, físico o social a los trabajadores de una organización. La carga de trabajo, los límites inexactos en las funciones del puesto, la falta de participación en la toma de decisiones, la inseguridad laboral y la falta de apoyo social, son algunos de los factores de riesgo psicosocial más representativos. Entre estos factores, la carga mental se presenta como el factor de riesgo psicosocial asociado a las características de la tarea más susceptible de provocar estrés laboral (Rubio, Díaz, Martín, \& Luceño, 2010). La carga mental surge como resultado de la dificultad del individuo para responder cuando las exigencias asociadas a las tareas son superiores a la capacidad del trabajador. El desequilibrio entre las demandas y las capacidades de la persona produce un incremento de la fatiga mental, una reducción de la atención, lentitud de pensamiento, mayor absentismo e, incluso, un incremento en el número de errores, olvidos y confusiones, que aumentan la probabilidad de que ocurran accidentes laborales. En este sentido, el estrés laboral se muestra como una de las principales consecuencias de la exposición prolongada a situaciones de excesiva carga mental, ya que el estrés, como dicen Moreno y Báez (2010), surge como resultado del balance que el sujeto hace entre las exigencias del puesto y su capacidad de afrontamiento. Por otra parte como consecuencia de las situaciones mantenidas de estrés, puede aparecer el burnout, o síndrome de quemarse en el trabajo (Albanesi de Nasetta, \& Morales de Barbenza, 2002; Bakker \& Demerouti, 2014).

La VII Encuesta de Condiciones de Trabajo realizada en España en 2011, señala que los riesgos psicosociales en el trabajo son la principal causa de enfermedades y accidentes laborales, con una prevalencia del $75 \%$ en el personal sanitario. En este sentido, el Instituto Nacional de Seguridad e Higiene en el Trabajo (INSHT), organismo dependiente del Ministerio de Trabajo español, ha señalado que las principales causas de los accidentes laborales se debían a distracciones, descuidos, despistes o falta de atención en un $45 \%$ de los casos, a trabajar demasiado rápido un $19,4 \%$, y al cansancio o fatiga en el $17,8 \%$, aspectos todos ellos directamente relacionados con la carga mental (Almodóvar, Pinilla, Galiana, \& Hervás, 2011). Atender a este tipo de situaciones en el ámbito sanitario tiene el doble de importancia dadas las graves consecuencias que tendrían un error o un accidente laboral dentro de este sector, no solo para el profesional, sino también para sus pacientes.

Cuixart y Cuixart (1990) señalan que los puestos asistenciales, como es el caso del trabajo hospitalario, son los más susceptibles de sufrir carga mental debido al tipo de trabajo que realizan estos profesionales. El profesional sanitario está sometido al mantenimiento elevado y constante de atención, lo que provoca continuas situaciones de sobrecarga. La información de la que disponen cambia rápidamente, siendo necesario un nivel mayor de concentración. Por último, la gravedad de las consecuencias de una toma de decisiones incorrecta, suma a estas condiciones de tensión una gran responsabilidad. Esto, añadido a la existencia de situaciones de incertidumbre, presiones temporales, los cambios de horario y el propio contacto con pacientes y familiares, hacen a los profesionales sanitarios muy vulnerables al estrés laboral (Gil-Monte, 2003, 2009; Reséndiz \& Reyes, 2012), llegando a ser clasificados como trabajadores que realizan una "profesión de alto riesgo psicosocial”. Más, Escribà y Cárdenas (1999), realizaron en un estudio cualitativo para 
determinar qué estresores laborales eran percibidos por el personal de enfermería hospitalario como más importantes. Para ello formaron dos grupos de discusión, constituidos por enfermeras y auxiliares de enfermería respectivamente, que pertenecían a diferentes servicios y hospitales. Ambos grupos señalaron como principales estresores laborales la falta de personal y la sobrecarga de trabajo. Otra serie de estresores que se detectaron fueron: los conflictos de jerarquía médico-enfermera/o-auxiliar de enfermería, las relaciones con pacientes y familiares, la infravaloración del trabajo realizado y los aspectos físicos del lugar de trabajo. Estos autores concluyeron que el personal de enfermería está expuesto a numerosos estresores, muchos de ellos específicos de las tareas de enfermería, y otros, relacionados fundamentalmente con la organización y las condiciones de trabajo, y que por tanto podrían evitarse.

A pesar de que los estudios sobre la carga mental en los profesionales sanitarios es amplia, son muy pocos los trabajos de investigación que se han dirigido a analizar las dimensiones o fuentes de carga mental que más les afectan. Un ejemplo es el estudio realizado por González, Moreno, Garrosa y López (2005), en el cual se analizó la asociación entre algunos de los factores laborales y demográficos y el nivel de carga mental y de fatiga laboral en una muestra de 228 enfermeros. Sus resultados mostraron que la dimensión que más afectaba era la demanda mental, seguida de la demanda física y la temporal respectivamente.

El objetivo de este trabajo es ahondar en el estudio de las diferentes fuentes de carga mental que presentan una muestra de enfermeros y técnicos auxiliares de enfermería de la Comunidad de Madrid en relación con una serie de funciones previamente definidas. Poder delimitar la influencia de cada una de las fuentes de carga mental permitirá ser más precisos en la localización de las necesidades de los trabajadores $y$ en el establecimiento de medidas de intervención más eficaces. Este trabajo aporta principalmente como novedad, a nivel español e iberoamericano, la utilización de la escala NASATLX para evaluar las fuentes de carga mental distinguiendo entre cada una de las funciones laborales del personal de enfermería, lo cual permite un diagnóstico más preciso de las mismas.

\section{Método}

\section{Participantes}

En este estudio se utilizó una muestra incidental formada por 297 participantes, todos ellos pertenecientes a un hospital público de la Comunidad de Madrid. Las categorías profesionales incluidas en el estudio fueron enfermeros $(n=166)$ y auxiliares de enfermería $(n=131)$. El $82.5 \%$ de los enfermeros y el $94.7 \%$ de los auxiliares eran mujeres, siendo un $87.9 \%$ de la muestra total mujeres y el $12.1 \%$ restante varones. La media de edad de los enfermeros era de 37.25 años (DT=.69) y de los auxiliares de 43.51 (DT=.93), siendo la media de la edad de la muestra total de 40.21 (DT=9.54). La antigüedad media en el puesto de trabajo de los enfermeros era de 13.12 años (DT=.65) y de los auxiliares de 15.54 años (DT=.94), siendo la antigüedad media en el puesto para la muestra total de 14.56 (DT=8.98).

El 58.7\% de los participantes estaba casado o vivía en pareja (59.3\% de los enfermeros y $55.6 \%$ de los auxiliares) y el $9.5 \%$ era viudo o estaba separado/divorciado (7.4\% de los enfermeros y $22.2 \%$ de los auxiliares). El $33.8 \%$ de la muestra total tenía entre 1 y 4 hijos, siendo lo más frecuente tener 2 hijos (52.2\%).

\section{Instrumento}

Para llevar a cabo el análisis de la carga mental se empleó la escala NASA-TLX (Task Load Index, Hart, \& Staveland, 1988). El NASA TLX es un procedimiento de valoración multidimensional que proporciona una puntuación global de carga de trabajo, obtenida a partir de la media de las puntuaciones en seis subescalas.

Las dimensiones que componen el NASATLX son: la demanda mental (actividad mental y perceptiva); la demanda física (grado de esfuerzo físico); la demanda temporal (sensación de presión temporal); el rendimiento (grado de cumplimiento de los objetivos); el esfuerzo (cantidad de esfuerzo físico y mental) y nivel de frustración (sensación de nerviosismo, desánimo, inseguridad, etc. durante la realización de la 
tarea). Según el modelo de Hart y Staveland (1988) que dio lugar a esta escala, las dimensiones demanda mental, física y temporal, son variables referidas a las demandas impuestas por el trabajo a la persona, mientras que las otras tres (esfuerzo, frustración y rendimiento) son el fruto de la interacción de la persona con la tarea.

La escala NASA-TLX se ha mostrado sensible a las variaciones intra y entre tareas, con una alta capacidad de diagnóstico de las fuentes de carga y relativamente insensible a las variaciones interpersonales (Arquel \& Nogareda, 2001). Las propiedades psicométricas del instrumento NASA-TLX han sido validadas por múltiples estudios comparativos de medida subjetiva de la carga mental (Hoonakker, Carayon, Gurses, Brown, Khunlertkit, McGuire, \& Walker, 2011; Rubio, Díaz, \& Martín, 2001; Rubio, Díaz, Martín, \& Puente, 2004), lo que unido a la sencillez de su aplicación lo hacen uno de los instrumentos más utilizados para medir la carga mental de trabajo (González, Moreno, Garrosa, \& López, 2005).

\section{Procedimiento}

En primer lugar, el equipo contactó con los responsables del servicio de prevención de riesgos laborales con el fin de obtener la aprobación para la aplicación del estudio. Estos responsables informaron a la plantilla de trabajadores sobre la naturaleza del estudio que se iba a realizar, explicando el carácter completamente anónimo y voluntario del mismo. A la muestra resultante se le administró la escala NASA-TLX con el fin de obtener una estimación acerca de los niveles de carga mental de las funciones de los referidos puestos de trabajo. Estas funciones fueron establecidas mediante reuniones entre los autores del estudio y los responsables del servicio de prevención. En la tabla 1 se presentan las cuatro funciones generales establecidas y sus respectivas definiciones para cada uno de los dos grupos profesionales, enfermeros y técnicos auxiliares de enfermería.

\section{Resultados}

Todos los análisis de los datos se realizaron con el programa estadístico SPSS. En primer lugar se analizaron mediante un análisis de varianza las posibles diferencias entre ambos puestos en sus valoraciones de carga mental global para cada una de las cuatro funciones. No se encontraron diferencias estadísticamente significativas en ninguno de los casos ( $p>.05$ ), por lo que ambos grupos de trabajadores fueron tratados como uno sólo, al que nos referiremos a partir de ahora como profesionales de enfermería.

Con el objetivo de analizar los niveles de carga mental global de las diferentes funciones y de conocer el grado en el que interviene cada una de las dimensiones de carga se realizaron diversos análisis descriptivos basados en el cálculo de la media y la desviación típica. Los resultados de estos análisis aparecen en las tablas 2 y 3.

Los resultados muestran que las funciones asistencial y administrativa son las que más carga global produce a estos trabajadores, seguidas de las funciones inherentes a la especialidad y de asistencia a facultativos (Tabla 2). Con el objetivo de analizar la significación estadística de las diferencias entre los niveles de carga global de las diferentes funciones, se realizó un análisis de comparación de medias mediante el estadístico $t$ de Student para muestras relacionadas. El resultado de este análisis mostró solo una diferencia significativa: la resultante de comparar la carga global de la función asistencial y la de la asistencia a facultativos $(\mathrm{t}=4.16, p=.000)(p>.05$ en el resto de comparaciones).

En cuanto a los resultados por dimensiones de carga para cada función, reflejan que la demanda mental y la temporal son las fuentes de carga de mayor peso para todas y cada una de las cuatro funciones (Tabla 3 )

$\mathrm{El}$ análisis de las dimensiones muestra una marcada tendencia estable a señalar la demanda mental y la demanda temporal como las fuentes de carga más importantes. Cabe destacar, por otro lado, las bajas puntuaciones obtenidas en frustración, especialmente en los referido a las funciones propias de la especialidad y a la función asistencial, así como los altos niveles de rendimiento alcanzados en estas mismas funciones, las cuales tienen un importante carácter vocacional.

Con el objetivo de analizar la significación estadística de las diferencias entre las dimensiones de carga para cada una de las funciones, se 
Tabla 1. Descripción de las funciones de los puestos de enfermero y de técnico auxiliar de enfermería

Funciones

Función asistencial

Función administrativa

Asistencia a otros facultativos

Funciones inherentes a la especialidad

\section{Enfermeros}

Encaminada a apoyar al paciente en su recuperación. Todas las tareas destinadas a los cuidados más básicos del paciente: recepción, movilización y asistencia básica médica y psiquiátrica del paciente, administración y preparación de medicamentos y tratamientos, información del estado de salud al paciente y a sus familiares.

Todas aquellas tareas relativas a la gestión documental, desde la realización de la historia clínica, registros y valoraciones del paciente, tanto inicial y durante el proceso de ingreso y alta, así como coordinación y asistencia a reuniones de equipo.

Incluye todas las tareas que precisen de acompañamiento a facultativos, ya sea participar en las visitas médicas, como prestar apoyo en la gestión de medicamentos, tareas de enfermería circulante y labores de asistencia en quirófanos.

Compuesta por el conjunto de tareas que no puedan ser agrupadas en los grupos anteriores por su carácter específico a la peculiaridad de la especialidad médica: como realizar anestesias u otras pruebas pertinentes, preparación de sala y material preciso para la intervención.
Técnicos auxiliares de enfermería

Atención y cuidados más básicos que recibe el paciente, desde la alimentación

a los traslados del mismo, incluso el acompañamiento y la administración de algún medicamento.

Tareas de almacenamiento, registro y reposición de medicamentos y materiales del almacén, funciones de preparación, limpieza y control del instrumental, equipos y maquinaria utilizada por los facultativos, así como registros oportunos de pacientes.

Referido a todas las tareas que precisen de acompañamiento a facultativos y a personal de enfermería.

Compuesta por el conjunto de funciones que no puedan ser agrupadas en los grupos anteriores por su carácter específico a la peculiaridad de la especialidad médica, como realizar asistencias en quirófanos.
Tabla 2. Estadísticos descriptivos de la carga global por funciones

\begin{tabular}{lcc}
\hline \multicolumn{1}{c}{ Funciones } & Media & $\begin{array}{c}\text { Desviación } \\
\text { Típica }\end{array}$ \\
\hline Función asistencial & 60.71 & 20.76 \\
Función administrativa & 59.53 & 19.36 \\
Asistencia a facultativos & 55.91 & 27.26 \\
Funciones de la especialidad & 57.51 & 21.46 \\
\hline
\end{tabular}

realizaron análisis de comparación de las medias mediante el estadístico $t$ de Student para muestras relacionadas. El resultado de estos análisis se muestra en la Tabla 4. Como puede observarse, gran parte de estas comparaciones resultaron significativas, especialmente al comparar las dimensiones rendimiento y frustración.

\section{Discusión y Conclusiones}

Los resultados de este estudio muestran diferencias en la carga mental que producen las diferentes funciones y dimensiones examinadas. La función asistencial es la que produce una mayor carga mental, seguida de la función administrativa y de aquella que incluye tareas inherentes a la especialidad. En último lugar, se encuentra la asistencia a facultativos, función que produce menor carga mental.

En cuanto al análisis detallado de las dimensiones consideradas, los resultados van en la línea de los obtenidos por Más, Escribá y Cárdenas (1999), quienes mediante un estudio cualitativo determinaron que la demanda mental 
Tabla 3. Estadísticos descriptivos de carga mental de cada dimensión para cada una de las funciones

\begin{tabular}{ccccccccc}
\hline \multirow{2}{*}{ Dimensiones } & \multicolumn{2}{c}{$\begin{array}{c}\text { Función } \\
\text { asistencial }\end{array}$} & \multicolumn{2}{c}{$\begin{array}{c}\text { Función } \\
\text { administrativa }\end{array}$} & \multicolumn{2}{c}{$\begin{array}{c}\text { Asistencia a } \\
\text { otros } \\
\text { facultativos }\end{array}$} & \multicolumn{2}{c}{$\begin{array}{c}\text { Funciones } \\
\text { inherentes a la } \\
\text { especialidad }\end{array}$} \\
\cline { 2 - 10 } & Media & D.T. & Media & D.T. & Media & D.T. & Media & D.T. \\
\hline Esfuerzo & 65.26 & 28.24 & 65.54 & 25.84 & 61.71 & 35.04 & 62.89 & 30.86 \\
Demanda Mental & 64.59 & 27.20 & 65.22 & 27.41 & 59.14 & 35.47 & 61.20 & 30.50 \\
Demanda Física & 56.52 & 27.99 & 49.84 & 29.60 & 44.18 & 33.12 & 53.14 & 30.26 \\
Demanda Temporal & 61.21 & 28.49 & 59.07 & 26.34 & 54.68 & 32.28 & 59.05 & 31.25 \\
Rendimiento & 80.35 & 21.23 & 77.26 & 21.99 & 70.15 & 31.93 & 82.24 & 18.73 \\
Frustración & 30.70 & 28.33 & 36.77 & 31.05 & 40.97 & 33.24 & 26.31 & 25.86 \\
\hline
\end{tabular}

Tabla 4. Resultados de los análisis de comparación de medias entre las dimensiones de carga para cada función

\begin{tabular}{|c|c|c|c|c|c|c|c|c|}
\hline \multirow[t]{2}{*}{ Dimensiones } & \multicolumn{2}{|c|}{$\begin{array}{c}\text { Función } \\
\text { asistencial }\end{array}$} & \multicolumn{2}{|c|}{$\begin{array}{c}\text { Función } \\
\text { administrativa }\end{array}$} & \multicolumn{2}{|c|}{$\begin{array}{l}\text { Asistencia } \\
\text { a otros } \\
\text { facultativos }\end{array}$} & \multicolumn{2}{|c|}{$\begin{array}{c}\text { Funciones } \\
\text { inherentes } \\
\text { a la } \\
\text { especialidad }\end{array}$} \\
\hline & $\mathrm{t}$ & sig. & $\mathrm{t}$ & sig. & $\mathrm{t}$ & sig. & $\mathrm{t}$ & sig. \\
\hline Esfuerzo-D. Mental & 0.32 & .743 & 0.19 & .846 & 0.91 & .363 & 0.85 & .393 \\
\hline Esfuerzo-D. Física & 6.40 & .000 & 8.72 & .000 & 6.26 & .000 & 4.34 & .000 \\
\hline Esfuerzo-D. Temporal & 2.16 & .032 & 4.06 & .000 & 3.04 & .003 & 1.37 & .173 \\
\hline Esfuerzo-Rendimiento & -8.72 & .000 & -5.77 & .000 & -2.52 & .013 & -6.00 & .000 \\
\hline Esfuerzo-Frustración & 16.74 & .000 & 11.62 & .000 & 5.48 & .000 & 11.75 & .000 \\
\hline D. Mental- D. Física & 5.39 & .000 & 7.52 & .000 & 5.09 & .000 & 4.15 & .000 \\
\hline D. Mental-D. Temporal & 2.41 & .017 & 3.64 & .000 & 1.83 & .070 & 1.00 & .320 \\
\hline D. Mental-Rendimiento & -8.83 & .000 & -5.53 & .000 & -3.07 & .003 & -7.10 & .000 \\
\hline D. Mental-Frustración & 16.13 & .000 & 10.82 & .000 & 4.96 & .000 & 11.15 & .000 \\
\hline D. Física-D. Temporal & -3.27 & .001 & -5.51 & .000 & -3.38 & .001 & -3.67 & .000 \\
\hline D. Física-Rendimiento & -13.26 & .000 & -13.32 & .000 & -7.15 & .000 & -9.25 & .000 \\
\hline D. Física-Frustración & 12.07 & .000 & 4.46 & .000 & 0.62 & .533 & 8.85 & .000 \\
\hline D. Temporal-Rendimiento & -10.33 & .000 & -9.81 & .000 & -4.49 & .000 & -7.83 & .000 \\
\hline D. Temporal-Frustración & 14.41 & .000 & 9.73 & .000 & 3.73 & .000 & 10.68 & .000 \\
\hline Rendimiento-Frustración & 21.81 & .000 & 15.46 & .000 & 6.99 & .000 & 18.42 & .000 \\
\hline
\end{tabular}

era el estresor laboral que el personal de enfermería percibía como más importante. De forma similar, Díaz, Rubio, Martín y Luceño (2010), en un estudio dirigido a analizar las características psicométricas del NASA-TLX con trabajadores españoles, mostraron que para los profesionales sanitarios una de las dimensiones más importante era la demanda mental. Más recientemente, trabajos como el de Hudson, Kushniruk y Borycki (2015), confirman, utilizando también la escala NASA-TLX, que las dimensiones de demanda mental y demanda temporal son las fuentes de carga con más peso en profesionales sanitarios.

La demanda temporal resultó ser la segunda dimensión de carga mental en orden de importancia en la muestra de profesionales de la enfermería que participaron en el estudio. Según los resultados de Más, Escribá y Cárdenas (1999), la falta de personal es percibida por los profesionales del rubro como el principal estresor laboral junto con la sobrecarga de trabajo, que 
constituye una consecuencia de la primera. Como sugieren Llop-Gironés, Tarafa y Benach (2015), el recorte del gasto sanitario y la falta de recursos que ha tenido lugar en España en estos últimos años, han traído como consecuencia una importante escasez de personal de enfermería en los centros sanitarios actuales, que ha dado lugar a graves problemas tanto en la calidad asistencial como en la reducción de la salud, física y psicológica, de este colectivo de profesionales. En este sentido, Crespo (2012) señala la importancia del recurso tiempo en relación con bienestar laboral en las organizaciones sanitarias. En su estudio, realizado con profesionales sanitarios españoles, mostró como la escasez de recursos humanos produce en los sujetos una presión asistencial que desemboca finalmente en una notoria sobrecarga de trabajo. Paralelo a este proceso, se produce un deterioro en las relaciones sociales creando malestar laboral, insatisfacción, baja autoeficacia y sentimientos de disminución de apoyo social por parte de la organización. Ante la necesidad de atender a los problemas que surgen de la demanda temporal, Crespo (2012) propone un aumento de los recursos humanos con el fin de contrarrestar el problema del tiempo y de la presión asistencial y de los efectos que ambas tienen en la organización y en sus usuarios. Insiste en la necesidad de una óptima gestión del tiempo para obtener un equipo eficaz, que genere un ambiente social positivo y de este modo mejorar el clima organizacional.

Resulta interesante la discordancia que, frente a dimensiones como la mental o la temporal, muestran los resultados en la dimensión frustración. Recordemos que dicha dimensión hace referencia a la sensación de presión, desánimo e inseguridad que presenta el trabajador durante la realización de la tarea. Mientras que la función asistencial resultó ser la que produce mayor carga mental, su nivel de frustración fue el más bajo. Este hecho se debe probablemente al carácter vocacional de este tipo de trabajo, a la gratificación que se produce con el contacto más humano y representativo de esta profesión. Diversos estudios plantean cómo los profesionales de la enfermería muestran algunas de las características de personalidad que actúan como moduladores en procesos de estrés. En este sentido, Roger y Abalo (2014), demuestran la estrecha relación que existe entre variables de influencia moduladora, como el sentido de la coherencia o la personalidad resistente, y la resistencia a los procesos de estrés laboral en una muestra de enfermeros cubanos. Asimismo, la función de asistencia a los facultativos señalada por los participantes como la que les produce menor carga mental, es la que muestra las puntuaciones más altas en frustración. Este hecho se ensambla con los problemas mencionados en la literatura, referidos a las dificultades en las relaciones con el resto de profesionales sanitarios. El estudio realizado por Más, Escribá y Cárdenas en 1999, ya mostraba este inconveniente como uno de los principales estresares laborales percibidos por el personal de enfermería. Se origen se encuentra, según los autores, en la falta de coordinación en la realización de las tareas propias del equipo. En los enfermeros debido a que su trabajo depende en cierto modo de la organización del facultativo especialista, y cuando se trata de los auxiliares por una delimitación poco clara entre sus funciones y las de los enfermeros. En ambos casos, esta problemática se traduce en conflictos dentro de la plantilla y en sentimientos de insatisfacción con el propio rol.

La carga mental refleja el coste humano que supone cumplir con las exigencias que requiere la realización de una tarea. Este coste puede ser puntual, de manera que el trabajador puede adaptarse a ese nivel de exigencia, o convertirse en un estado continuo de activación. En este último caso, la carga mental supone un estado de vulnerabilidad a la fatiga y a sufrir algún tipo de desequilibrio en su salud tanto física como psicológica. El diseño adecuado de las condiciones de trabajo, de las características y exigencias propias de la tarea, resulta crucial para prevenir situaciones de peligro para los trabajadores. En el caso de los profesionales sanitarios, correr este tipo de riesgos puede tener graves consecuencias además sobre la salud de las personas bajo su cuidado. Responder a las necesidades de evaluación e intervención de los profesionales sanitarios supone una inversión de futuro para garantizar la salud y el bienestar de los trabajadores y, con ello, la calidad del sistema de salud, de ahí la necesidad de seguir profundizando 
en el análisis y diagnóstico de las condiciones de trabajo del personal dedicado a "profesiones de alto riesgo" como es el caso del sector enfermería.

Nuestro estudio ha permitido analizar las funciones laborales que para los profesionales de la enfermería suponen una mayor fuente de carga mental, así como ha aportado mayor evidencia acerca de las dimensiones de carga más importantes en este colectivo profesional. De igual forma, nuestros resultados pueden ser útiles a la hora de compararlos con los encontrados en otras investigaciones futuras. Sin embargo, no está exento de limitaciones que deben tenerse en cuenta al diseñar nuevas investigaciones en este campo. Una de ellas se relaciona con el uso de una muestra incidental, lo cual limita la capacidad de generalización de los resultados. En este sentido sería recomendable poder disponer de un muestreo aleatorio de los participantes, así como de trabajadores de diferentes hospitales, centros de salud, lugares geográficos, etc. De igual forma sería muy conveniente poder realizar estudios de carácter longitudinal que permitan establecer la estabilidad temporal de las evaluaciones así como evaluar la eficacia de posibles programas de intervención dirigidos a la reducción de los niveles de carga del personal de enfermería.

\section{Referencias}

Albanesi de Nasetta, S., \& Morales de Barbenza, C. (2002). Burnout en el personal de enfermería de un hospital. Revista Iberoamericana de Diagnóstico y Evaluación - e Avaliação Psicológica, 14, 87-101.

Almodóvar, A., Pinilla, F. J., Galiana, L., \& Hervás, P. (2011). Exposición a riesgos en el trabajo. Principales resultados de la VII Encuesta Nacional de Condiciones de Trabajo. Madrid: INSHT.

Arquer, I., \& Nogareda, C. (2001). NTP 544: Estimación de la carga mental de trabajo: El método NASA TLX. Barcelona: INSHT.

Bakker A. B., \& Demerouti, E. (2014). Job demands-resources theory. En P.Y. Chen y C. Cooper (eds.), Wellbeing: A Complete Reference Guide, Work and Wellbeing (Vol. 3) (pp. 37-64). Chichester: UK: John Wiley \& Sons.
Crespo, J. L. (2012). El recurso tiempo para el bienestar laboral en la organización sanitaria saludable. Póster presentado en el I Congreso Nacional de Psicología Positiva. El Escorial. España.

Cuixart, C. N., \& Cuixart, S. N. (1990). Valoración de la carga mental en el servicio de urgencias de un hospital. Salud y Trabajo, 82, 11-15.

Díaz, E., Rubio, S., Martín, J., \& Luceño, L. (2010). Estudio psicométrico del índice de carga mental NASA-TLX con una muestra de trabajadores españoles. Revista de Psicología del Trabajo y de las Organizaciones, 26(3), 191-199.

Gil-Monte, P. (2003). El síndrome de quemarse por el trabajo (Síndrome de Burnout) en profesionales de enfermería. Revista Eletrônica InterAção Psy, 1, 19-33.

Gil-Monte, P. (2009). Algunas razones para considerar los riesgos psicosociales en el trabajo y sus consecuencias en la salud pública. Revista Española de Salud Pública, 83(2), 169-173.

González, J. L., Moreno, B., Garrosa, E., \& López, A. (2005). Carga mental y fatiga en servicios especiales de enfermería. Revista Latinoamericana de Psicología, 37(3), 477492.

Hart, S. G., \& Staveland, L. E. (1988). Development of NASA-TLX (Task Load Index): Results of empirical and theoretical research. Advances in Psychology, 52, 139183.

Hoonakker, P., Carayon, P., Gurses, A. P., Brown, R., Khunlertkit, A., McGuire, K., \& Walker, J. M. (2011). Measuring workload of ICU nurses with a questionnaire survey: The NASA task load index (TLX). IIE transactions on Healthcare Systems Engineering, 1(2), 131-143.

Hudson, D., Kushniruk, A. W. \& Borycki, E. M. (2015). Using the NASA task load index to assess workload in electronic medical records. Driving Quality in Informatics: Fulfilling the Promise, 208, 190-194.

Llop-Gironés, A., Tarafa, G. y Benach, J. (2015). Personal de enfermería, condiciones de 
trabajo y su impacto en la salud. Gaceta Sanitaria, 29(2), 152-157.

Más, R., Escribà, V., \& Cárdenas, M. (1999). Estresores laborales percibidos por el personal de enfermería hospitalario: Un estudio cualitativo. Archivos de Prevención de Riesgos Laborales, 2(4), 159-167.

Moreno, B., \& Báez, C. (2010). Factores y riesgos psicosociales: Formas, consecuencias, medidas y buenas prácticas. Madrid: UAM.

Reséndiz, A., \& Reyes, I. (2012). Validación de una lista de chequeo de estresores con intensidad para médicos residentes. Revista Iberoamericana de Diagnóstico y Evaluación - e Avaliação Psicológica, 33, 111-125.

Roger, M. C., \& Abalo, J. A. G. (2014). Burnout y variables personales moduladoras en enfermeros que trabajan en hospitales oncológicos. Psicología y Salud, 14(1), 67-78.

Rubio, S., Díaz, E., Martín, J., \& Luceño, L. (2010). La carga mental como factor de riesgo psicosocial. Diferencias por baja laboral. Ansiedad y Estrés, 16(2-3), 271-282.

Rubio, S., Díaz, E., \& Martín, J. (2001). Aspectos metodológicos de la evaluación subjetiva de la carga mental de trabajo. Archivos de Prevención de Riesgos Laborales, 4(4), 160168.

Rubio, S., Díaz, E., Martín, J., \& Puente, J. M. (2004). Evaluation of subjective mental workload: A comparison of SWAT, NASATLX, and workload profile methods. Applied Psychology, 53(1), 61-86.

Sauter, S. L., Murphy, L. R., Hurrell, J. J., \& Levi, L. (2001). Factores psicosociales y de organización. OIT. Enciclopedia de Salud y Seguridad en el Trabajo, vol II, cap. 34. Madrid: Ministerio de Trabajo y Asuntos Sociales. 